\title{
Observation and Measurement of Temperature Rise and Distribution on GaAs Photo-cathode Wafer with a 532nm Drive Laser and a Thermal Imaging Camera
}

\author{
S. Zhang, S.V.Benson, and C. H-Garcia \\ Thomas Jefferson National Accelerator Facility, Newport News, VA 23606, USA
}

\begin{abstract}
Significant temperature rise and gradient are observed from a GaAs photo-cathode wafer irradiated at various power levels with over $20 \mathrm{~W}$ laser power at $532 \mathrm{~nm}$ wavelength. The laser power absorption and dissipated thermal distribution are measured. The result shows a clear indication that proper removal of laser induced heat from the cathode needs to be considered seriously when designing a high average current or low quantum efficiency photocathode electron gun. The measurement method presented here provides a useful way to obtain information about both temperature and thermal profiles, it also applies to cathode heating study with other heating devices such as electrical heaters.
\end{abstract}

Keywords: Electron Sources, Beam injection and extraction, Design of specific laser system. PACS: 29.25.Bx, 29.27.Ac, 42.60.By

\section{INTRODUCTION}

The high-voltage DC gun with a photo-cathode has been a very important electron source for generating high-average-current short electron bunches. The quantumefficiency $(\mathrm{QE})$ of a GaAs photo-cathode can be much higher than other types of cathodes such as metals. A well-processed Negative Electron Affinity (NEA) GaAs wafer can usually provide a few percent $\mathrm{QE}$ at the desired laser wavelength. A very successful example is the injector for Jefferson Lab's ERL accelerator, which uses a $10 \mathrm{~mA}$ electron gun with a cesiated GaAs photo-cathode [1]. With a given QE, the amount of needed laser power increases proportionally with the expected electron current and photon energy. Driven and demanded by the development of new generation light sources, photo-cathode guns with much higher average current have recently been proposed and are under study in different labs [2]. A typical and durable QE during operation is about $1 \%$. This requires $234 \mathrm{~mW} / \mathrm{mA}$ of laser power for a 532 $\mathrm{nm}$ laser wavelength. Lower QEs or shorter wavelengths require even higher power.

With the rapid development of the advanced laser technology, the laser power is no longer a limiting factor. However, it is not clear how much laser power the photo cathode can actually accept in terms of the thermal load without active cooling. It is known that a big portion of incident laser power will be absorbed by semiconductor wafer and turn into heat. The temperature on the cathode may increase significantly due to the limited thermal conductivity of the wafers and the improper heat transfer. 
One consequence from the heated cathode is the loss of the QE, which can be fatal to its application in high beam current machines. Tests have indicated the drop of $\mathrm{QE}$ scales up with the cathode temperature rise. There were very limited studies [3] reported before, it is apparently a very complicated subject and it is still not clear at the moment what the exact physical mechanism behind the phenomenon is. It is believed that thermal desorption of $\mathrm{Cs} / \mathrm{O}$ adlayer due to the temperature rise plays an important role in the degradation of the photo emission from NEA GaAs cathodes, even without any extraction of the electron beam from the surface. On one hand, there is no question that lots of careful studies are needed in order to understand the physics and chemical process involved. On the other hand, one important and obvious missing subject from the previous study is that only an electrical heater was employed to raise the cathode temperature, instead of using a laser beam at sufficient power level. So far there has been no detailed investigation on the cathode heating characteristics with a high power laser beam. We have performed such measurement with a real ERL drive laser and same cathode wafer used for ERL photocathode gun at Jlab FEL facility. The result revealed the significant temperature rise and gradient on the cathode, clearly indicating that proper removal of laser induced heat from the cathode needs to be considered seriously when designing a high average current or low quantum efficiency photo-cathode electron gun with similar cathode materials.

\section{DESCRIPTION OF THE EXPERIMENTAL SYSTEM}

The laser used for this experiment was designed as a drive laser for an ERL-based accelerator with beam current up to $100 \mathrm{~mA}$. The diode-pumped $\mathrm{Nd}: \mathrm{YVO}_{4}$ system with a master-oscillator-power-amplifier configuration provides over $25 \mathrm{~W}$ output power at $532 \mathrm{~nm}$ wavelength. Although the laser was mode-locked at $75 \mathrm{MHz}$ and each micro-pulse in the pulse train is $25 \mathrm{ps}$ long, the heating depends only on the average power due to the high repetition rate. The sample wafer is a 1 inch GaAs square, the same as what has been mounted in JLab FEL photocathode gun for ERL machine operation. The thickness of the wafer is $1 \mathrm{~mm}$. The wafer is heat sunk but not cooled for the measurement reported in this paper. A layer of indium foil is placed between the wafer and the surface of a flat aluminum alloy base mount to keep them in good thermal contact. The air-conditioned room temperature is about $23^{\circ} \mathrm{C}$. It should be pointed out that in actual injector use the cathode would be in vacuum and temperature rise could be slightly higher. Depending on the specific accelerator configurations and their applications, various laser beam sizes on cathodes have been taken at different facilities, ranging from less than $1 \mathrm{~mm}$ up to $10 \mathrm{~mm}$. To take a medium size, we expanded the laser beam so that the laser spot on the wafer surface is about 5mm (FWHM) with a Gaussian distribution. The absorbed laser power in the wafer is obtained by measuring both the input and the reflected laser power with power meters. It is easy to imagine the thermal distribution induced by laser heating will not be uniform even if the laser beam is an ideal flat-top shape. It is equally important to measure the thermal distribution rather than just an average temperature over the cathode area because the temperature gradient will cause mechanical stress which may lead to further degradation of the photo cathode performance. In order to 
know both the temperature and its distribution on the wafer surface, a thermal imaging camera (FLIR-A, manufactured by FLIR Systems) is used. This kind of infrared camera system provides an accurate solution especially for non-contact imaging and temperature measurements. We performed careful emissivity calibration on the camera before each measurement. The schematic and a picture for the experimental setup are shown in Figure 1. From Figure 1(b) a clear thermal image of the whole wafer assembly can be seen when the wafer is irradiated and heated by the laser beam. All the data is recorded and processed by a personal computer.

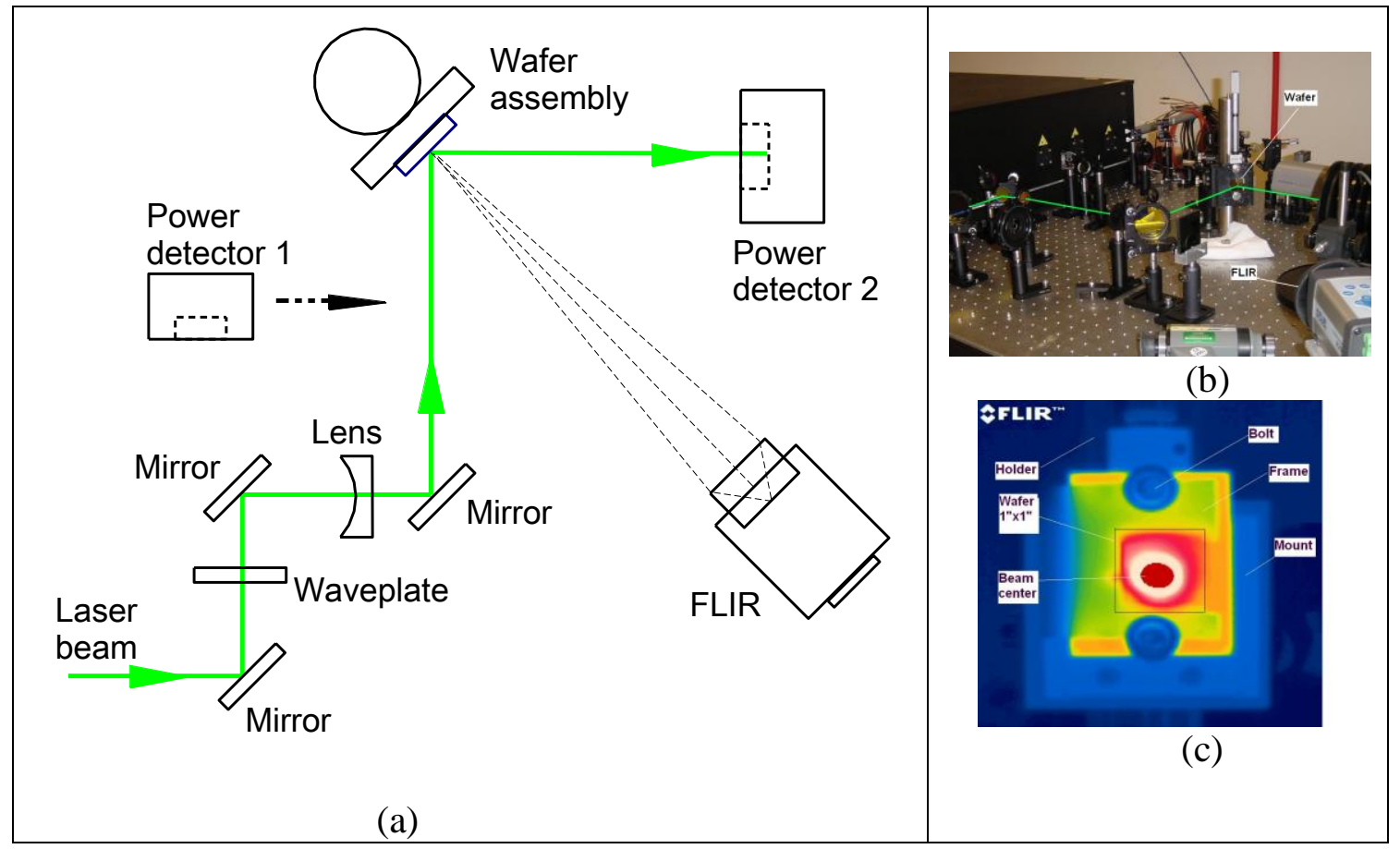

FIGURE 1. (a) Optical schematic of the experimental setup. (b) A picture of the system. (c) An image of the wafer assembly by IR (FLIR) camera when laser beam is on. A negative lens is used for beam expansion and a waveplate for change of polarization.

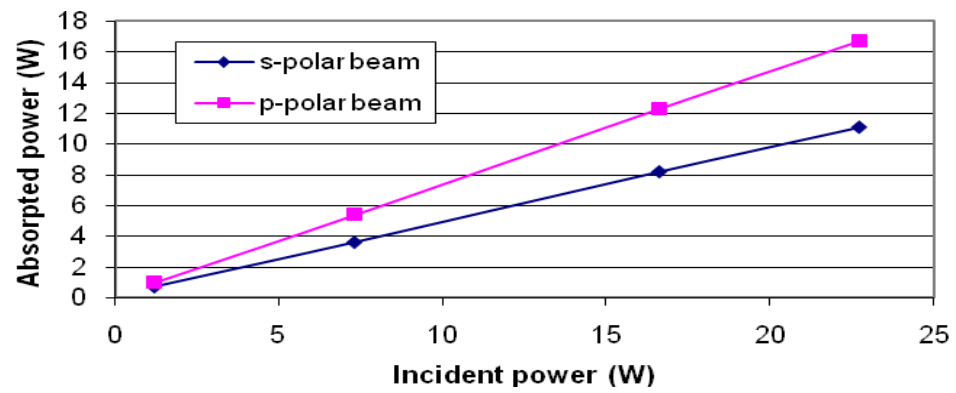

FIGURE 2. Measured laser power absorption by GaAs wafer at 532nm laser wavelength. The absorption is defined as the ratio of absorbed power over the incident power. Beam polarization sign: $\mathrm{s}$, vertical polarization. $\mathrm{p}$, horizontal polarization. 


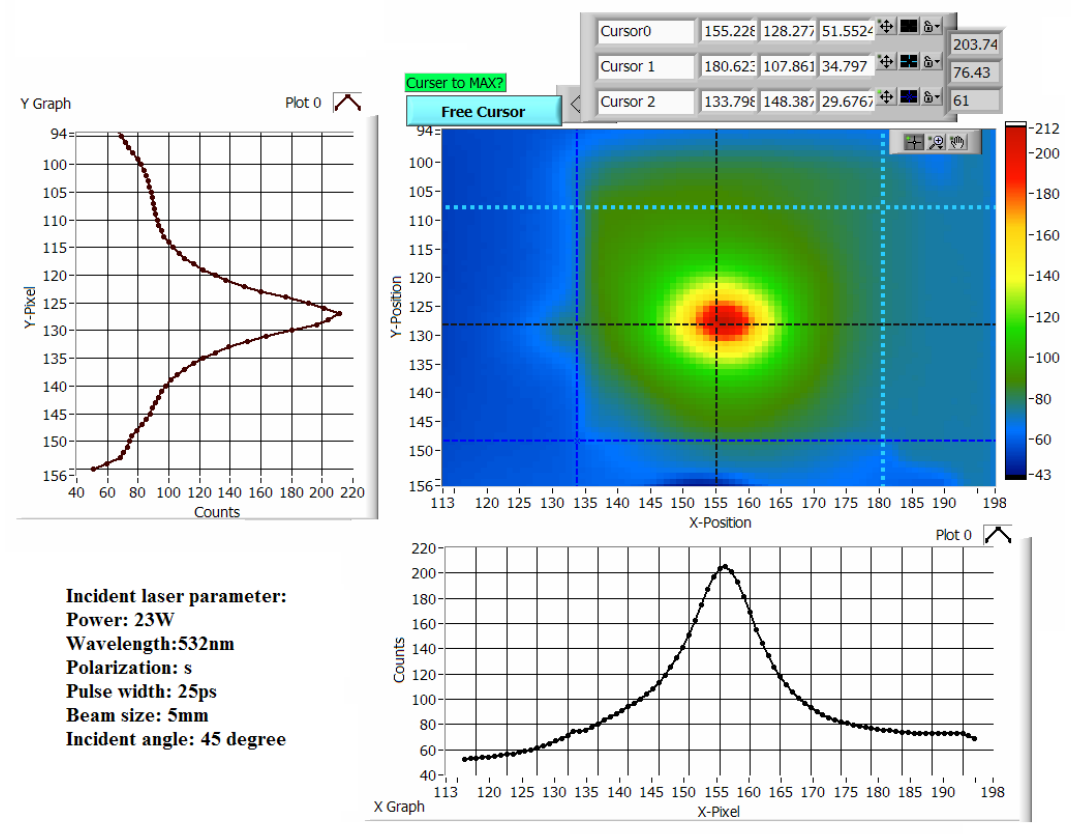

(a)

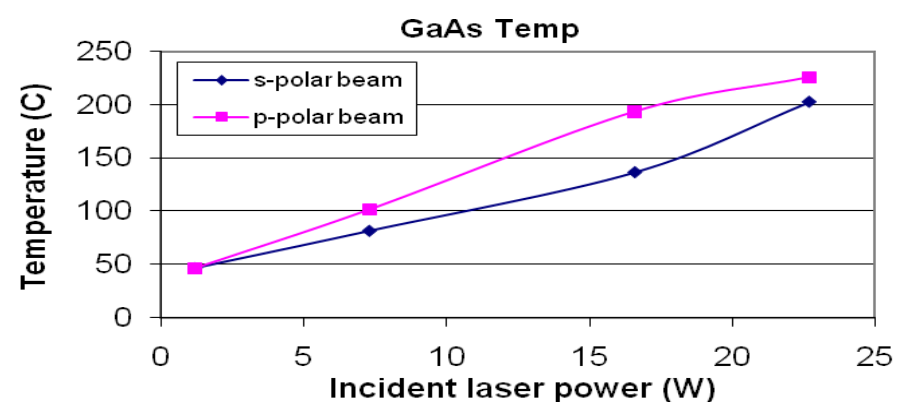

(b)

FIGURE 3. (a)Thermal image of the wafer and the temperature distribution under $23 \mathrm{~W}$ laser beam. (b)Dependence of temperature on the incident laser power. The ambient temperature is about $23^{\circ} \mathrm{C}$ degree.

\section{RESULTS AND DISCUSSION}

Like other optical materials, the reflectivity of the GaAs wafer is closely related to the polarization of the laser beam and its incident angle. We measured the reflectivity of GaAs wafer under test in order to calculate the actual absorption by wafers. As shown in Figure 2, at 45 degree angle the difference between a horizontally-polarized (p) beam and a vertically-polarized (s) is remarkable, the former is about $74 \%$ and the later $48 \%$. In other words, only $26 \%$ of the p-polarized input laser is reflected by the wafer while about $52 \%$ of s-polarized beam power goes to the beam dump. This suggests that a p-beam is preferred to reduce the laser power needed if a 45 degree incident angle has to be taken. At a near normal angle, there is little difference and the absorption is about $65 \%$ for both as expected. Based on the reflectivity, we can calculate the refraction index of the wafer at $2.33 \mathrm{eV}$ photon energy (or $532 \mathrm{~nm}$ ) is 
about 4.4. It is difficult to cross check this result with other resources since it is extremely scarce in literature. It should be pointed out that the roughness of the wafer surface will also affect the reflectivity and laser power measurement.

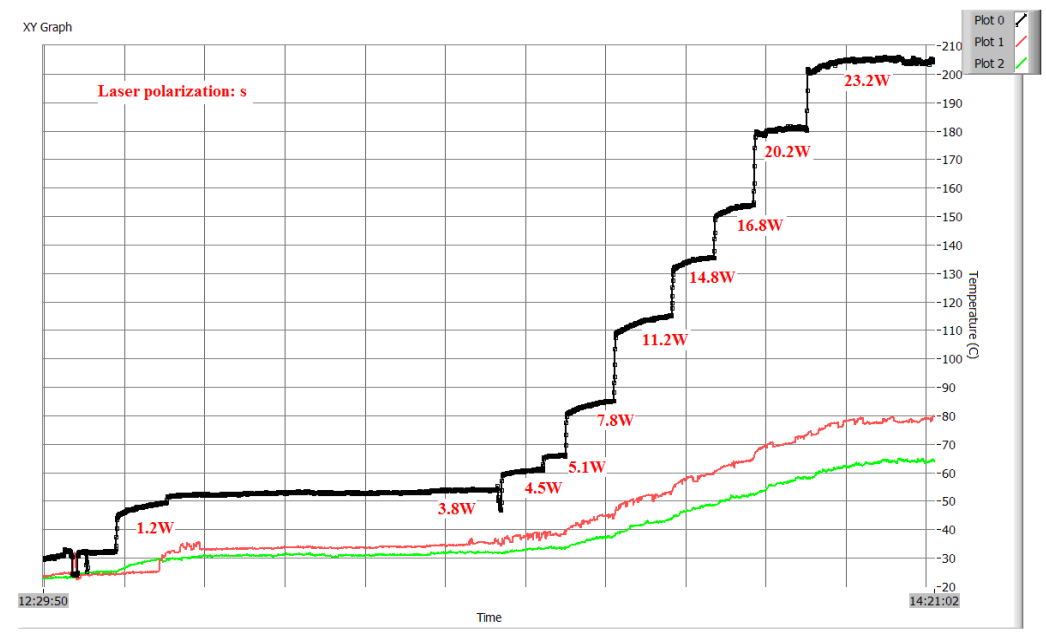

(a)

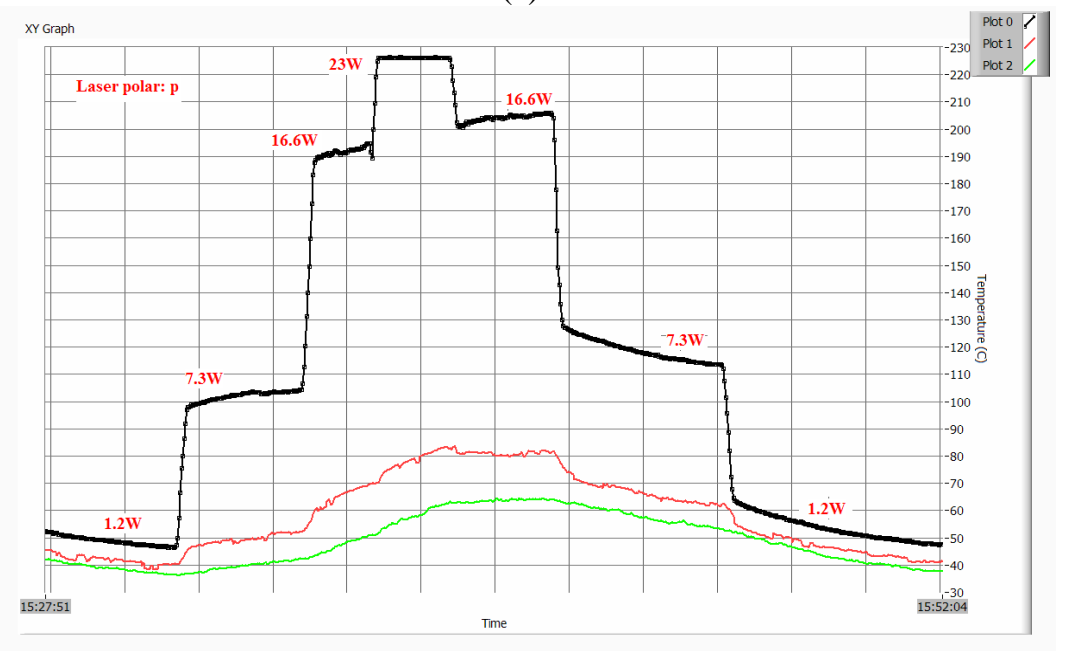

(b)

FIGURE 4. (a) Wafer temperature versus incident laser power with s-polarized beam. (b) Temperature trend when the laser power ramps up and down. The thick black curve refers to the center of laser beam position. The thin green and red curves are the temperatures at the cursors' position near wafer edges, as shown in the screen snap-shot in Figure 4(a).

When the wafer is irradiated with $23 \mathrm{~W}$ of laser power, the thermal distribution resembles a Gaussian-like shape, as shown in Figure 3(a). The center where laser hits the surface has the highest temperature, which is over $200{ }^{\circ} \mathrm{C}$. Even the edges of the square reach to $70{ }^{\circ} \mathrm{C}$. The ambient temperature has been kept at $23{ }^{\circ} \mathrm{C}$. A comparison between $\mathrm{p}$ and $\mathrm{s}$ polarized beams is shown in Figure 3(b). As expected, the wafer temperature with the p-beam is higher than that of the s-beam simply due to the higher heat load as indicated by the higher power absorption. From Figure 4, we can see the 
temperature follows fairly promptly the laser power change and tends to be stabilized at certain level in just a few minutes. Also it is interesting to see that the slower and smoother temperature trend shown by the thin red and blue curves for the position near the corners of the wafer. This means a delay and thermal equilibrium process happened when the heat goes from the center to the edge.

The shape of the thermal distribution on the wafer resembles that of the laser beam spot. The elliptical shape is due to the 45 incident angle which is also responsible for the relatively wider peak and slower ramp compared with that on the vertical axis. The thermal gradient is obviously not uniform cross the whole area. The temperature difference between the center and the edge of the wafer reaches $130^{\circ} \mathrm{C}$. Such a nonlinear, high temperature gradient may lead to severe mechanical stress and could eventually significantly affect the photo cathode performance. This is an important subject to study in future.

We can see the immediate fall-off of the temperature following the laser power drop from Figure 4(b), which indicates that the thermal contact between the wafer and the metal base is good since the heat is quickly dissipated into the base in spite of the known fact that GaAs is not a very good thermal conductor. Finally, we performed the same test on the same wafer after the indium foil was removed, in order to get an idea how well the heat was transferred away from the wafer through the indium foil. To our surprise, the wafer shattered within 2 minutes after the laser power reached $23 \mathrm{~W}$. In the result shown in Figure 5, the sudden fall-off of the black curve near the right end is when the shattering happened and the laser power went directly into the base.

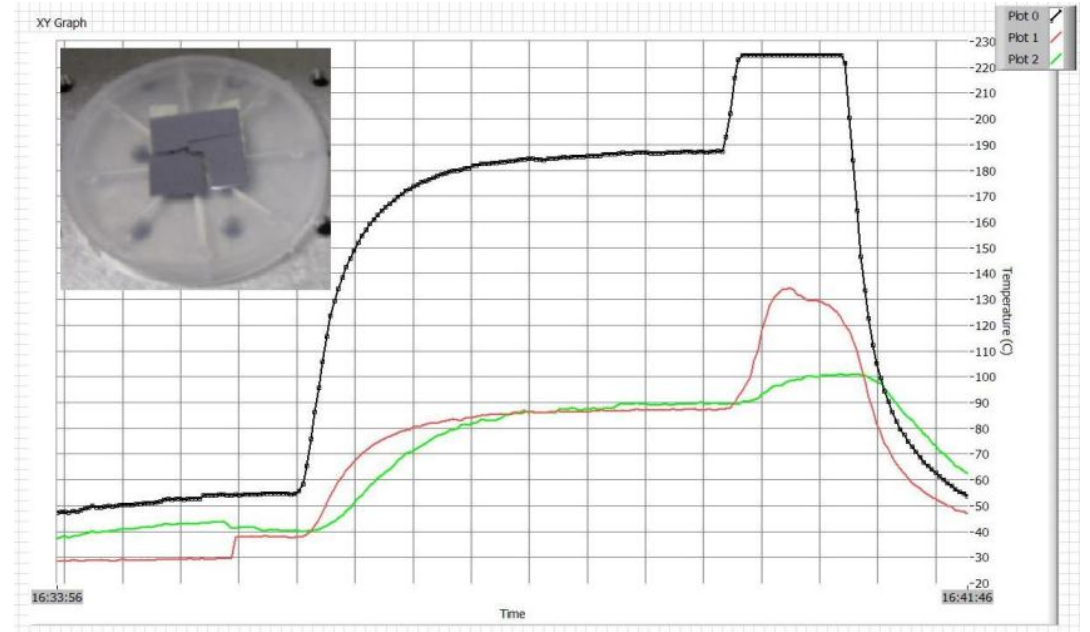

FIGURE 5. Temperature versus incident laser power without indium foil. The colors of the curves are the same as in Figure 5. The top-left inset is a picture of the shattered wafer on a plastic case cover.

\section{CONCLUSION}

The heating effect on the GaAs wafer due to laser power dissipation leads to significant temperature rise and gradient when irradiated by over $20 \mathrm{~W} 532 \mathrm{~nm}$ laser power. This imposes a serious issue for such types of photo-cathode gun, especially for those used at high current or low quantum efficiency, as even more laser power 
may be needed than reported here. Proper cooling measures have to be considered in the gun design. It is also important in future to study the mechanical stress induced by the large temperature gradient, how it affects the electron beam property and what is an acceptable temperature on the wafer. The measurement method presented here provides a useful way to obtain important information about both temperature and thermal distribution, including the heating study with other heater such as electrical heaters.

\section{ACKNOWLEDGMENTS}

The authors thank T. Powers for the kind assistance with the FLIR camera. We also thank M. Poelker and M. Stutzman for very helpful technical discussion. This work is supported by Office of Naval Research and DOE Contract DE-AC05-060R23171.

\section{REFERENCES}

1. C. Hernandez-Garcia, T. Siggins, S. Benson, D. Bullard, H. F. Dylla, K. Jordan, C. Murray, G. Neil, M. Shinn and R. Walker, "A High Average Current DC GaAs Photocathode Gun for ERLs and FELs", edited by C. Horak, Proceedings of 2005 Particle Accelerator Conference, Knoxville, TN, 2005, pp. 3117-3119.

2. C. B. M. Dunham, C. K. Sinclair, I. V. Bazarov, Y. Li, X. Liu, and K. W. Smolenski, "Performance of A Very High Voltage Photoemission Electron Gun for A High Average Current ERL Injector", Proceedings of PAC07, Albuquerque, New Mexico, 2007, pp.1224-1226.

3. M. Kuriki, et al., "Dark-lifetime degradation of GaAs photo-cathode at higher temperature", Nucl. Instr. and Meth. A (2010) (in press). 